\title{
Tuberculous pericarditis: An exceptional STEMI association: Case report
}

Among all the complications of myocardial infarction, pericardial effusion remains correlated with a high morbidity and mortality rate. Tuberculosis in Morocco is a public health problem with 25,000 new cases per year, $47 \%$ of which are extra pulmonary, we present an exceptional case of a 70 year old man presenting with an extensive anterior myocardial infarction complicated with tuberculous pericarditis effusion, highlighting the importance of thinking of
this aetiology in a specific context.

Keywords: Tuberculous pericarditis; STEMI

\section{Case Description}

A 70 year-old diabetic and hypertensive man with no family history of $\mathrm{TB}$ or chronic chest condition, was admitted within 4 hours of an extensive anterior ST elevation myocardial infarction (STEMI) (Figure 1). He was immediately transferred to the cathlab for primary percutaneous intervention. Coronary angiography analysis showed an occlusion of the mid left anterior descending artery (LAD) (Figure 2 ). The case was manged by stenting with predilatation with a satisfying result (Figure 3). Thransthoracic echocardiography (TTE) showed an akinesia in the territory of the LAD (apex, anteroseptal, anterior and anterolateral segments) with mild systolic left ventricle dysfunction. During the hosptalisation, we noted the onset of dyspnea and bilateral lower limb swelling of lower limb with no friction rub. Systematic TTE revealed small to moderate pericardial effusion but no sign of tamponade, with elevated left ventricular (LV) filling pressure. We decided to manage the case as myocardial infarction complicated by
Sanoussi H, Kourireche N, Lakhal Z, Raissuni M, Sabri M, Chaib A and Zbir M Department of Cardiology, Military Hospital Mohammed V of Rabat, Morocco *Author for correspondence: dr.hamzasanoussi@gmail.com Tel: +212678328407

congestive heart failure by salt restriction and diuretics. Patient showed clinical improvement of his condition and was discharged with a slight pericardial effusion, with a close follow-up in consultation. Three months later, the patient was seen in consultation for dypnea at rest with signs of right heart failure, fever, night profuse sweating and a weight loss amounts to 12 $\mathrm{kg}$. The chest radiography revealed a pleural effusion with cardiomegaly, without pericardial calcification. TTE showed a circumferential abundant pericardial effusion, without evidence of tamponade with a persistent mild systolic left ventricle dysfunction and normal (LV) filling pressure (Figure 4). Pericardial liquid analysis revealed positive QuantiFERON$\mathrm{TB}$ assay $(7.95 \mathrm{UI} / \mathrm{mL})$ and elevated Adenosine Desaminase level. Microscopic examination was not contributive. Serology showed negative HBV, HCV, HIV and syphilis. The patient was treated by ant tuberculosis chemotherapy. Successive TTE revealed the disappearance of the pericardial effusion after 2 months of treatment.
Submitted: 17 August 2017

Accepted: 19 September 2017

Published online: 25 September 2017

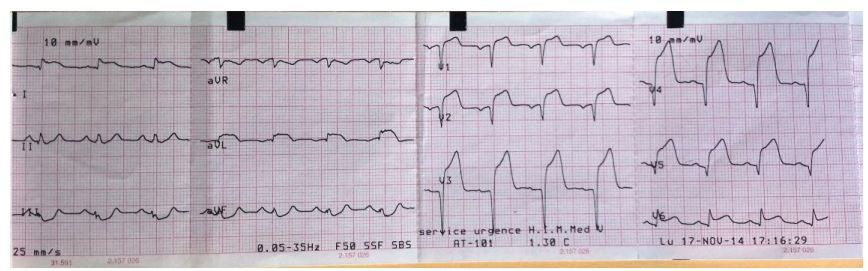

Figure 1: A 12-leads ECG showing extensive anterior STEMI. 


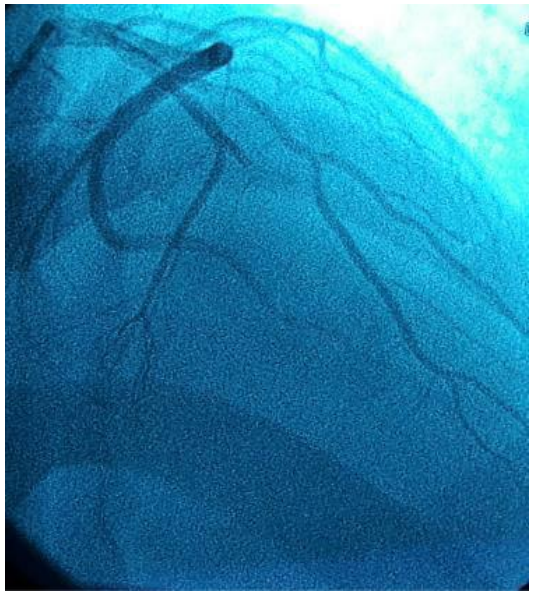

Figure 2: Coronary angiography showing an occlusion of the mid left artery descending (LAD).

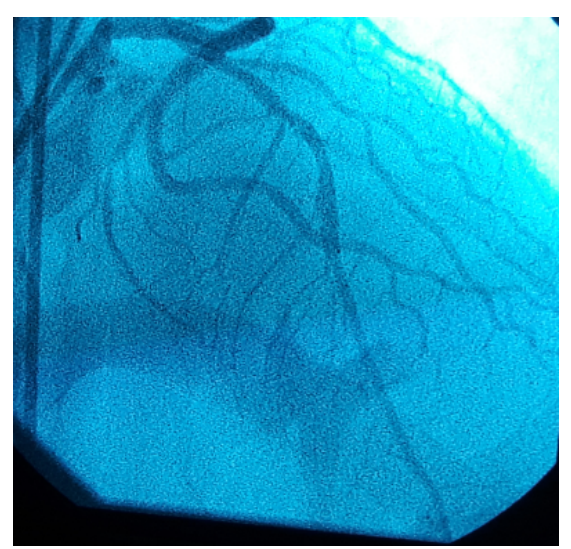

Figure 3: Successful angioplasty of the occluded LAD with the drug eluting stent deployed.

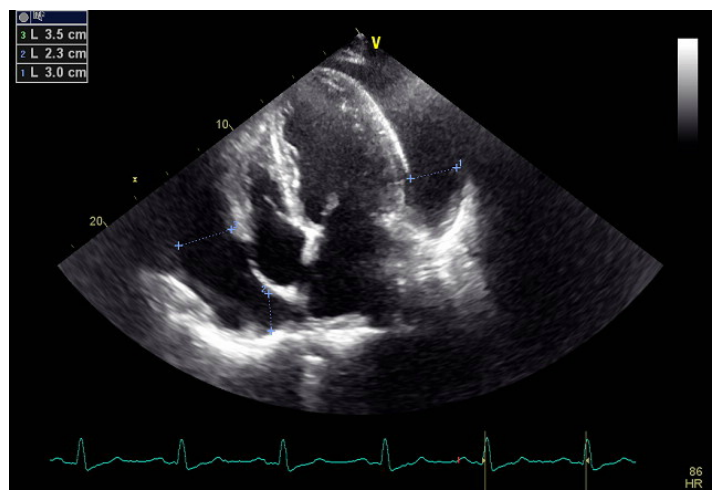

Figure 4: Apical view in transthoracic echocardiography showing the circumferential pericardial effusion with no tamponade sign.

\section{Discussion}

Tuberculosis (TB) is one of the most widespread infections in the world [1], and considered as public health problem with 9 million cases in the world in 2012 [1], and 27.000 new cases per year in Morocco, with a non-negligible prevalence of $14 \%$ of the extrapulmonary form [2]. Tuberculous pericarditis (TBP), remains one the main cause of hospitalization pericardial effusion and heart failure in developing countries [3]. Three forms of presentation of tuberculous pericarditis: The effusive form $(80 \%)$, the constrictive (5\%), and the effusive-constrictive pericarditis (15\%) [4]. Clinical presentation is heterogeneous. The symptomatology may variate from simple coughing to exertional dyspnea with congestive heart failure, orthopnea and lower limb swelling. Common symptoms may also include fever with night sweats, weight loss [5-7]. Signs of chronic right heart failure due to effusive cardiac compression remains the most common type of presentation [6,8]. Making the diagnosis of TBP relaying only on symptomatology remains a difficult task, indeed signs and symptoms are generally not specific the echocardiography is the gold standard to make a certain diagnosis of the pericardial effusion. Computed tomography (CT) and Magnetic resonance imaging (MRI) are more efficient but they may generally be missing in developing and poor countries [5-7]. The chest radiography may look for pulmonary lesions suggestive of active tuberculosis, pleural effusion and cardiomegaly $[9,10]$. In the majority of cases of TBP, the ECG is abnormal [11]. The modifications concerns usually the ST-T-wave but they remain nonspecific $[11,12]$. The ST and PRsegment variations are found in $10 \%$ to $12 \%$ of cases [12]. The presence of microvoltage has a good sensibility but is not specific of the pericardial effusion [12]. Less frequently transient atrial fibrillation occurs in $4 \%$ of cases, and electrical alternans which is more specific of swinging heart and cardiac tamponade [9]. All this electrical abnormalities can be hidden in case of ST elevation myocardial infarction (STEMI). The fibrinous strands as seen in TTE are not specific for a tuberculous etiology $[9,13]$.

There are two methods diagnosis of TBP: Direct and indirect methods: The Direct Method is Pericardiocentesis which is recommended every time TBP is suspected. Macroscopic analysis finds blood stained fluid in $80 \%$ of cases. Microscopic analysis shows typically exudative fluid with high protein content, cytology is remarkable for a predominance of monocytes and lymphocytes count. Light's criteria [14] remain the most accurate way to identify pericardial exudates effusions [15]. Anatomopathology of the pericardial biopsy specimen make certitude diagnosis of $\mathrm{TB}$, but thoracotomy and all its complications with increased morbidity and marked prolongation of the hospital stay may limit the procedure [16]. However the sensitivity diagnostic of pericardial biopsy for TP ranges only from $10 \%$ to $64 \%[17,18]$. In other more valuable diagnosis tool, we find the polymerase chain reaction (PCR) for detecting Mycobacterium tuberculosis DNA in pericardial effusion fluid [19]. In tuberculous endemic countries, the high incidence of $\mathrm{TB}$, the probability of cross-sensitization from mycobacterium, and mass 
BCG vaccination, make tuberculin skin testing not very useful [20].

The other method of TBP diagnosis is the indirect one: Dosing the Adenosine desaminase (ADA) activity in pericardial liquid can make the diagnostic of TBP as showed in several studies [21-23]. Elevated ADA activity ranging from 30 to $60 \mathrm{U} / \mathrm{L}$ is suggestive of tuberculosis with a specificity and sensitivity diagnosis rate of $74 \%$ and $90 \%$ [23]. High levels of the ADA are known to be a reliable prognostic indicator for the development of constrictive pericarditis in TBP. Pericardial lysozyme is also described as a diagnostic tool for TBP. Cutoff levels of $6.5 \mu \mathrm{g} / \mathrm{dL}$ has been described to make the diagnosis of TBP with a sensitivity and specificity rates of $100 \%$ and $91 \%$ respectively [22]. Elevated interferon- $\gamma$ (IFN- $\gamma$ ) levels in pericardial liquid can be used in early diagnosis of TBP with a sensitivity and specificity rate respectively of $92 \%$ and $100 \%$ [22]. Being a rapid and highly specific test of TBP diagnosis, the IFN- $\gamma$ is now the test of choice for the diagnosis, but technical and financial constraints may limit its use in many developing countries [23].

Before the advent of antibiotics mortality was 80 to $90 \%$, with the antituberculosis chemotherapy, mortality rate decreased significantly [24]. The mortality rate ranges from $8 \%$ to $17 \%$ in HIVnegative patients $[25,26]$ and $17 \%$ to $34 \%$ in HIVpositive patients [27]. The antituberculosis protocol consists on a quadruple therapy of pyrazinamide, rifampicin, ethambutol, and isoniazid, for two months, followed by isoniazid and rifampicin for four months [28,29]. No data had shown advantages of lengthening treatment duration although for 9 months or longer, on the contrary some cases of treatment poor compliance was described [29]. The effectiveness of Short-course chemotherapy is also proved in TBP in HIV-positive patients [30]. Association of corticosteroids was described to be effective in constrictive form but remains controversial in the effusive form of tuberculous pericarditis [31].

\section{Conclusion}

Early pericardial effusion due to left ventricular free wall rupture and congestive heart failure are the main causes for pericardial effusion post-ST-elevation MyocardiaI infarction (MI). It is important to think about tuberculosis etiology in a context of endemic countries.

\section{Executive Summary}

Among all the complications of myocardial infarction, pericardial effusion remains correlated with a high morbidity and mortality rate.

Tuberculosis in Morocco is a public health problem with 25,000 new cases per year, $47 \%$ of which are extra pulmonary.

We present an exceptional case of a 70 year old man presenting with an extensive anterior myocardial infarction complicated with tuberculous pericarditis effusion, highlighting the importance of thinking of this aetiology in a specific context.

\section{References}

1. World Health Organization. Global Tuberculosis Report, Geneva. (2013).

2. Galve E, Garcia-Del-Castillo H, Evangelista A, Batlle J, Permanyer-Miralda G, Soler-Soler J. Pericardial effusion in the course of myocardial infarction: incidence, natural history, and clinical relevance. Circulation. 73: 294-299 (1986).

3. Mayosi BM, Wiysonge CS, Ntsekhe M, et al. Mortality in patients treated for tuberculous pericarditis in sub-Saharan Africa. S. Afr. Med. J. 98: 36-40 (2008).

4. Strang JIG. Tuberculous pericarditis-Clinical Medicine and Health in Developing Africa. Cape Town. David. Philip. Press. pp: 145-149 (1982).

5. Hageman JH, d'Esopo ND, Glenn WWL. Tuberculosis of the pericardium: a long-term analysis of forty-four proved cases. N. Engl. J. Med. 270: 327-332 (1964).

6. Desai HN. Tuberculous pericarditis: a review of 100 cases. $S$. Afr. Med. J. 55: 877- 880 (1979).

7. Fowler NO, Manitas GT. Infectious pericarditis. Prog. Cardiovasc. Dis. 16: 323 (1973).

8. Strang JIG. Tuberculous pericarditis in Transkei. Clin. Cardiol. 7: 667-670 (1984).
9. Commerford PJ, Strang JIG. Tuberculous pericarditisACentury of Tuberculosis: South African Perspectives. Cape Town: Oxford. University. Press. pp: 123-136 (1991).

10. Sagrista-Sauleda J, Permanyer-Miralda G, Soler-Soler J. Tuberculous pericarditis: ten-year experience with a prospective protocol for diagnosis and treatment. J. Am. Coll. Cardiol. 11: 724-728 (1988).

11. Smedema JP, Katjitae I, Reuter $\mathrm{H}$, et al. Twelve lead electrocardiography in tuberculous pericarditis. Cardiovasc. J. S. Afr. 12: 31-34 (2001).

12. Smedema JP, Katjitae I, Reuter H, et al. Twelve lead electrocardiography. Cardiovasc. J. S. Afr. 12: 31-34 (1999).

13. Liu PY, Li YH, Tsai WC, Chao TH, Yung YJ, Chen JH. Usefulness of echocardiographic intrapericardial abnormalities in the diagnosis of tuberculous pericardial effusion. Am. J. Cardiol. 87: 1133-1135 (2001).

14. Light RW. Pleural effusions. Med. Clin. North. Am. 61: 1339-1352 (1977).

15. Burgess LJ, Reuter H, Carstens ME, Taljaard JJ, Doubell AF. Cytokine production in patients with tuberculous pericarditis. Int. J. Tuberc. Lung. Dis. 6: 439-446 (2002).

16. Louw VJ, Reuter H, Smedema JP, Katijitae I, Burgess L, Doubell AF. Clinical experience with pericardiocentesis and 
extended drainage in a population with a high prevalence of HIV. Neth. Heart. J. 10: 399-406 (2002).

17. Schepers GWH. Tuberculous pericarditis. Am. J. Cardiol. 9: 248-276 (1962).

18. Komsouglu B, Goldeli O, Kulan K, Komsouglu SS. The diagnostic and prognostic value of adenosine deaminase in tuberculous pericarditis. Eur. Heart. J. 16: 1126-1130 (1995).

19. Brisson-Noël A, Gicquel B, Le Coissier D, Lévy-Frébault V, Nassif X, Hance AJ. Rapid diagnosis of tuberculosis by amplification of mycobacterial DNA in clinical samples. Lancet. 2: 1069-1071 (1989).

20. Ng TTC, Strang JIG, Wilkins EGL. Serodiagnosis of pericardial tuberculosis. Q. J. Med. 88: 317-320 (1995).

21. Latouf SE, Levetan BN, Commerford PJ. Tuberculous pericardial effusion: analysis of commonly used diagnostic methods. S. Afr. Med. J. 86: 15 (1996).

22. Aggeli C, Pitsavos C, Brili S, et al. Relevance of adenosine deaminase and lysozyme measurements in the diagnosis of tuberculous pericarditis. Cardiology. 94: 81-85 (2000).

23. Burgess LJ, Reuter H, Carstens ME, Taljaard JJF, Doubell AF. The use of adenosine deaminase and interferon-gamma as diagnostic tools for tuberculous pericarditis. Chest. 122: 900905 (2002).

24. Harvey AM, Whitehill MR. Tuberculous pericarditis. Medicine. 16: 45-94 (1937)

25. Gooi HC, Smith JM. Tuberculous pericarditis in Birmingham. Thorax. 33: 94-96 (1978).

26. Bhan GL. Tuberculous pericarditis. J. Infect. 2: 360-364 (1980).

27. Hakim JG, Ternouth I, Mushangi E, Siziya S, Robertson V, Malin A. Double blind randomised placebo controlled trial of adjunctive prednisolone in the treatment of effusive tuberculous pericarditis in HIV seropositive patients. Heart. 84: 183-188 (2000)

28. Cohn DL, Catlin BJ, Peterson KL, Judson FN, Sbarbaro JA. A 62-dose, 6-month therapy for pulmonary and extrapulmonary tuberculosis: a twice-weekly directlyobserved, cost-effective regimen. Ann. Intern. Med. 112: 407415 (1990).

29. Combs DL, O'Brien RJ, Geiter LJ. USPHS Tuberculosis Short-Course Chemotherapy Trial 21: effectiveness, toxicity and acceptability: the report of final results. Ann. Intern. Med. 112: 397-406 (1990)

30. Perriens JH, St Louis ME, Mukadi YB, et al. Pulmonary tuberculosis in HIV infected patients in Zaire: a controlled trial of treatment for either 6 or 12 months. N. Engl. J. Med. 332: 779-785 (1995).

31. Ntsekhe M, Wiysonge C, Volmink JA, Commerford PJ, Mayosi BM. Adjuvant corticosteroids for tuberculous pericarditis: promising, but not proven. Q. J. Med. 96: 593599 (2003). 